ARTÍ́CULO

DE REFLEXIÓN

\title{
LA CONFIANZA, LOS VALORES Y EL CAPITAL SOCIAL EN JOVENES ... Hacia una compresión de la trascendencia humana
}

\author{
THE TRUST, THE VALUES AND THE SOCIAL CAPITAL IN YOUNG. \\ ... Toward an understanding of the human transcendence.
}

Nhora Lucía Aguirre Aricapa ${ }^{1}$

${ }^{1}$ Universidad de Manizales, Liceo Arquidiocesano de Nuestra Señora, Manizales- Colombia. nolua@hotmail.com.

Recibido: Enero 18 de 2012

Aceptado: Abril 17 de 2012

*Correspondencia del autor. Universidad de Manizales, Caldas- Colombia. nolua@hotmail.com.

\section{RESUMEN}

Los jóvenes se agrupan y se reúnen con base en objetivos concretos, cuando se trata de expresar solidaridad y presencia ante la vulneración de los derechos de los demás. Es allí donde se evidencia el capital social y sus posibilidades para contribuir a apuntalar proyectos y ayudar a la sociedad en su búsqueda de mayor equidad y justicia social.

De ahí que Fukuyama en su libro, la gran ruptura(1999), plantea que "los valores éticos le abren paso al capital social"; ya que a partir de relaciones más profundas, éstas asociaciones generan ganancia no solo para una persona, sino para un grupo o una comunidad.

Palabras claves: Capital social, valores, confianza, asociacionismo, ciudadanía, jóvenes.

\begin{abstract}
The youth's group and they meet with base in concrete objectives, when it is to express solidarity and he/she witnesses before the vulneration of the rights of the other ones. It is there where it is evidenced the social capital and their possibilities to contribute to bolster projects and to help to the society in their search of more justness and social justice.

With the result that Fukuyama in their book, the great rupture (1999), outline that "the ethical values open step to the social capital"; since starting from the deepest relationships, these associations generate gain, not alone for a person, but for a group or a community.
\end{abstract}

Key words: Social capital, value, trust, asociacionismo, citizenship. 
A fines de la década de los setentas y durante los ochenta, las principales teorizaciones sobre la noción de capital social corresponden a Bourdieu (1980). Desde la sociología de la cultura, sistematiza el concepto a partir de un abordaje fundamentalmente instrumental, centrándose en los beneficios que reciben los individuos en función de su participación en grupos y en la construcción deliberada de la sociabilidad con el objetivo de crear ese recurso.

En los últimos años ha crecido el interés por el estudio de las consecuencias positivas del capital social, al considerarlo como el conjunto de relaciones sociales de las que en un determinado momento dispone un sujeto individual. Al realizar el desplazamiento del nivel individual al colectivo se aprecian las redes como la base del capital social, según Coleman (1990) y así mismo relacionarlo con "la capacidad de los individuos de trabajar junto a otros, en grupos u organizaciones, para alcanzar objetivos comunes".

Los jóvenes se agrupan y se reúnen con base en objetivos concretos, cuando se trata de expresar solidaridad y presencia ante la vulneración de los derechos de los demás e incluso para participar en procesos que involucre metas de mayor calado idealista, como en el caso de luchar por la paz. Es allí donde se evidencia el capital social y sus posibilidades para contribuir a apuntalar proyectos a las personas y ayudar a la sociedad en su búsqueda de mayor equidad y justicia social.

Se observa en esos atisbos de capital social un entrelazamiento de las relaciones sociales y diferentes valores que refuerzan y apuntalan la construcción de capital social y con los cuales se dinamizan las acciones o comportamientos de los individuos en el grupo.

Para Putnam (1994) "capital social es el conformado por el grado de confianza existente entre los actores sociales de una sociedad, las normas de comportamiento cívico practicadas y el nivel de asociatividad que la caracteriza, elementos éstos que evidencian la riqueza y la fortaleza del tejido social interno de una sociedad. Es un sistema de redes, normas y la confianza, que facilita la coordinación y cooperación para el beneficio mutuo".

La confianza ocupa un papel preponderante, toda vez que se constituye en esencial en las relaciones con el otro, consigo mismo y con su espacio social, al proporcionarle seguridad en su saber y hacer, y proporcionarle sostenibilidad a la forma particular como se haya asu- mido la asociatividad.

Francis Fukuyama (1996) ve el capital social como "la capacidad que nace a partir del predominio de la confianza, en una sociedad o en determinados sectores de ella".

No solo en grupos de interés como serían los jóvenes sino también en las empresas, instituciones comunitarias u otros grupos donde se asocien y luchen por un bien común, dando prioridad nuevamente al elemento de la confianza como uno de los valores más relevantes en la relación con otros.

Kenneth Newton (1997) expresa que "el capital social puede ser visto como un fenómeno subjetivo, compuesto de valores y actitudes que influyen en como las personas se relacionan entre sí. Incluye confianza, normas de reciprocidad, actitudes y valores que ayudan a las personas a trascender relaciones conflictivas y competitivas para conformar relaciones de cooperación y ayuda mutua".

Propone así, en su definición de capital social, como los valores que cada persona vivencia en su cotidiano hacen que se acerque y se relacione al otro, menciona la confianza dentro de esa gama de valores sociales como uno de los más sobresalientes y sin la cual no se podría llegar a tener capital social.

Stephan Baas (1997) alude a que capital social "tiene que ver con cohesión social, con identificación con las normas de gobierno, con expresiones culturales y comportamientos sociales que hacen a la sociedad más cohesiva y algo más que una suma de individuos".

Al referirse a expresiones culturales y comportamientos sociales hace un acercamiento a los valores que son los que se viven al interior de las comunidades, le dan identidad al grupo y a una comunidad, transmitiendo éste grupo de códigos, generación tras generación.

James Joseph (1998) dice que "es un vasto conjunto de ideas, ideales, instituciones y arreglos sociales, a través de los cuales las personas encuentran su voz y movilizan sus energías particulares por causas públicas”.

En éste concepto de capital social se visualiza en realidad un componente individual que hace que un grupo se fortalezca a partir del individuo, cuando habla de arreglos sociales son encuentros entre las personas, los cuales se pueden hacer a partir de los valores, ideales 
o ideas que cada uno desee exponer y ponerlos a favor del grupo.

Para Bullen y Onyx (1998,) capital social "implica redes sociales, basadas en principios de confianza, reciprocidad y normas de acción".

De nuevo, la confianza dentro del grupo, genera capital social, puesto que implica un proceso de reciprocidad, sin el cual no se da, así mismo se remite a normas de acción o bien llamados códigos éticos o cívicos, los cuales hacen que sea posible la comprensión y tolerancia para alcanzar unos objetivos comunes.

Don Cohen y Laurence Prusak (2001) señalan que capital social "es el conjunto de todas las conexiones activas entre la gente: la confianza, la comprensión mutua y los valores y conductas compartidas que vinculan a los miembros de las redes y comunidades humanas y que hace posible una acción cooperativa".

Una vez más los valores tienen un papel importante en cuanto a las relaciones entre los seres humanos y cómo éstos generan un ambiente importante donde se puede accionar y llevar a cabalidad cualquier objetivo que conduzca a la ganancia para todos y no solo para una persona.

Dentro de ese entramado es bueno señalar que se encuentra un elemento básico para que esas relaciones se den, es ahí, donde el código ético y/o moral se hace notar con más fuerza; ya que de él dependen las relaciones que emprenda el ser humano con otro ser humano. De ahí que Fukuyama en su libro, la gran ruptura(1999), plantee que "los valores éticos le abren paso al capital social"; ya que a partir de un saludo, unas gracias o simplemente el darse cuenta de los gustos de otra persona son como mínimo reglas de cortesía que abre caminos hacia relaciones más profundas, que redundan en intereses y asociaciones que generan de una $\mathrm{u}$ otra forma ganancia no sólo para una persona sino para un grupo o una comunidad.

\section{La Búsqueda Valoral}

Durante todo el desarrollo del ser humano desde sus etapas iniciales, es necesario brindarle a esa criatura información acerca del cómo se está transformando el mundo que lo rodea y cómo se va transformando él mismo, al habitar y hacer parte de ese todo que lo rodea.

Es innegable que el ser humano así como estudia y co- noce ese espacio que ve y observa, también tiene una tarea muy ardua; de conocerse así mismo, de saber bajo qué formas, instancias, normas rige su propio destino, rige su vida, así mismo debe conocer el cómo es que se relaciona con los otros congéneres y éstos a su vez qué redes pueden llegar a conformar y hacer de éstas una verdadera riqueza, la cual controlaría las diferentes oportunidades de una persona, una familia, una sociedad, una región e incluso un país.

Es entonces en éste contexto donde los valores humanos cumplen con un rol sumamente importante al ser éstos los que rigen, direccionan la vida de cada ser en su comportamiento cotidiano y constituyen un puente teórico entre un enfoque meramente económico y un enfoque social y cultural del desarrollo de la sociedad. En esta indagación se busca dar cuenta de los valores más importantes en la construcción de las relaciones que los jóvenes de Manizales manejan, así mismo cómo el valor de la confianza permea el quehacer y el origen de esas relaciones, las cuales potencian a cada ser humano y hacen que se encuentren y se tomen oportunidades valiosas para competir en un mundo cada vez más rudo en el sostenimiento y sobrevivencia de la especie misma.

Los valores constituyen lineamientos intangibles que rigen todo comportamiento humano, de hecho, racionalizado, imaginado y/o como un acto creador. Hacen que el ser se sienta armónico y equilibrado cuando se es coherente en su ser, hacer, pensar y sentir, y al ser vivenciados de esa forma, logra que a nivel social se construya en positivo todos los alcances que ésta desee para sus miembros.

Su origen data de tiempos remotos y se conocían como virtudes. Han sido cultivados y practicados en todas las sociedades y culturas, desde las muy antiguas hasta las modernas, rigiendo los comportamientos humanos dentro de la libertad que éste tiene con la toma de decisiones en la primera institución social como es la familia, que es donde nacen, se desarrollan y crecen constantemente. No son estáticos como se pensaba inicialmente, son dinámicos y muestra de ello es la movilidad que han tenido en la última década, antes primaba, la vida, la justicia, el trabajo; actualmente los jóvenes señalan el respeto, la responsabilidad y la tolerancia.

Para Irrazabal (2009) "Los valores se caracterizan por ser fuerzas intrínsecas del espíritu del hombre, que se hacen extrínsecas en el medio social mediante el acto voluntario de la persona. Este es consentido por la so- 
ciedad cuando resulta positivo y beneficioso y rechazado, al ser negativo que es dañino para el hombre y la sociedad. Los valores positivos son fuerzas generadoras de progreso y engendran el desarrollo integral del hombre y de la sociedad, están encaminados a conseguir una dinámica social acompañada para llegar a la homologación del ser humano; única forma de alcanzar el bien común. Los valores cultivados por el hombre en forma positiva, afianzan su individualidad y su personalidad. Los antivalores se caracterizan porque producen caos, incomprensión e incapacidad para resolver los problemas que afectan a la sociedad"

Quienes viven de acuerdo con sus valores y hacen de éstos prácticas comunes en su diario vivir, están preparados para brindar y aportar al bienestar social; en otras palabras aprenden a contribuir con el bienestar de la sociedad.

Guiddens, ( 2001) hace relación a la necesidad de compromisos morales, que trasciendan las preocupaciones y riñas triviales de la vida diaria; entendiendo como compromiso moral las pautas que de una u otra forma le permite al ser humano construir una vida sólida y decidir con criterios adecuados, llevando a pensar, que es necesario tener esos lineamientos intangibles siempre presentes en todo momento de la vida.

Es así como los valores bien cimentados ayudan a que las personas sean de bien, a tener proyectos de vida profundos y los llevan a la búsqueda de la paz interior y por consiguiente a la paz en su familia, en su comunidad, en su sociedad.

Históricamente los valores cultivados por el ser humano en forma positiva, afianza su personalidad, su individualidad, su identidad; pero al mismo tiempo vemos reflejado en la actualidad grandes deseos de cooperar, de expresar solidaridad al otro, de apoyar y colaborar para que no solamente le vaya bien a un individuo, sino al grupo y por ende a la comunidad y a la sociedad de la cual se hace parte.

Es necesario tener claro que los valores se enseñan, se transmiten de generación en generación, pero cada uno los va introyectándo y los va poniendo a prueba al relacionarse con sus amigos, sus compañeros y sus familiares, dándoles su transformación propia.

El ser humano necesita desde la infancia que le hablen de la importancia del amor, del respeto por las diferencias, de la tolerancia y del verdadero valor del ser humano, cuando llega a la adolescencia una de sus tareas psíquicas principales son las de clasificar y aclarar su propia escala de valores, y evidenciarla en su proyecto de vida, en la toma de decisión vocacional.

Irrazabal (2009) presenta una clasificación de los valores:

Valores morales, guían y regulan los actos tales como la fortaleza, templanza, honorabilidad, modestia, humildad, sinceridad, rectitud y bondad.

Valores civiles, regulan el trato con el prójimo dentro de la convivencia social. En ellos figuran la confianza, la prudencia, veracidad, respeto, dignidad, generosidad y el altruismo. Es en ésta clase de valores donde el capital social se evidencia y trae con ellos desarrollo, unidos a los valores cívicos.

Valores cívicos, referidos al comportamiento con la patria tales como: lealtad, patriotismo, responsabilidad, coraje, cooperación, laboriosidad.

Valores personales, defienden la vida humana, preservándola del asecho que en su contra hacen las prácticas abortivas, la dictadura, el egoísmo, la miseria, matando la vida, la libertad, la justicia y la igualdad.

Valores universales, se cultivan en todas partes del mundo por ser esenciales para toda la humanidad tales como: el bien, la verdad, la justicia, la libertad, la igualdad y la paz. Estos le dan piso a las redes sociales que se están creando.

Actualmente, más que de valores en sí, se habla de los derechos humanos, los cuales son un conjunto de valores que obligan al respeto de la vida, a la integridad física e intelectual del hombre, a la libertad de creencia, al ejercicio de la justicia, de la verdad, de la tolerancia, de la solidaridad, de la igualdad.

Estos valores constituyen los más esenciales. Los valores de la persona humana requieren de un cultivo intenso a fin de devolverle al hombre su status y pueda con ello manejar realmente el alcance de sus objetivos positivos, de la sociedad en que se encuentre. "sólo la práctica de los buenos valores nos llevará al desarrollo personal y a conseguir un bien común”. (Irrazabal, 2009). Objetivos claros en la perspectiva del capital social.

Es bueno tener claro que cada una de las clases de valores nos lleva a conseguir objetivos individuales y también objetivos a nivel colectivo. 


\section{LA CONFIANZA Y EL CAPITAL SOCIAL}

Cuando Newton (1997) toma el capital social como fenómeno subjetivo compuesto por valores y actitudes que influyen en la forma como se relacionan las personas, incluye confianza, normas de reciprocidad, actitudes y valores que ayudan a la gente a superar relaciones conflictivas y competitivas para establecer lazos de cooperación y ayuda mutua (citado por Marcelo Martínez, 2000); lleva a comprender que los valores son aspectos y actitudes que las personas tienen en cuenta para relacionarse o para llegar a conformar grupos.

Para el capital social es fundamental los valores cívicos y los civiles; ya que éstos regulan los comportamientos individuales en función de un colectivo, allí se encuentran la confianza como dador de seguridad y compromiso, el respeto, la lealtad, la responsabilidad, la cooperación y la laboriosidad. Valores éstos que cultivados a nivel personal proporcionan al ser humano elementos básicos para entrar a relacionarse y a cualificarse con los otros, son el abre- caminos en esas relaciones sociales que más adelante redundan en desarrollo de objetivos comunes a un grupo o comunidad.

La confianza es para Guiddens (1996), un aspecto fundamental para el desarrollo de la personalidad y para la potenciación de rasgos distintivos y particulares en un mundo de mecanismos desmembradores y sistemas abstractos. Esto hace referencia directa a un cierto sentido de seguridad ontológica. La confianza establecida entre el niño y sus padres le suministra un escudo que lo protege contra potenciales amenazas y peligros de la vida cotidiana; ella aísla los acontecimientos que de ser contemplados en toda su magnitud, producirían trastornos en la voluntad o sensaciones de abatimiento que alteran el estilo de vida.

El estilo de vida es crucial en la constitución de la identidad y en la actividad diaria y se materializa en la toma de decisiones de los sujetos.

Si el estilo de vida de un individuo está impregnado de confianza, este podrá interiorizar al otro y al mundo y establecerá relaciones abiertas y sinceras, con los demás y con su entorno, pues la confianza puede ser movilizada, únicamente, por un proceso de apertura mutua que presupone el compromiso de un impacto positivo sobre el otro y sobre el mundo.

Fukuyama (1999) plantea que para que un país pueda adaptarse correctamente a un mundo globalizado, es fundamental que su gente tenga confianza en las instituciones.

Si ésta no existe o es escasa, las redes sociales se ven resquebrajadas, por consiguiente la lucha por un bien común se afecta, pues no se va a buscar apoyo a ellas, al no creer que puedan ser una base para conseguir objetivos comunes.

Plantea igualmente que esas redes de confianza generan un grado esperado de transparencia, al establecer una relación de confianza, luego se podrá llegar a una relación de negocios.

Es de esperar que los jóvenes deseen creer en las instituciones, tener la seguridad que a través de ellas pueden alcanzar sus sueños, pero al confiar poco, como lo indican los resultados de esta investigación, solo refleja la credibilidad que ellos están proyectando, lo cual conduce a pensar en nuevas estrategias para que el joven se acerque, conozca y re-conozca en ellas una buena fuente de solidaridad y apoyo social.

Así mismo, las instituciones deben pensar en el cómo hacer que los jóvenes confíen en ellas y es a través de la generación de relaciones, que al interior de las mismas se vivencie: transparencia, respeto y lealtad.

La confianza está fuertemente ligada a la creatividad, ya que la confianza es en sí misma creativa y trae consigo un compromiso que le permite a la persona dar un salto a lo desconocido, un asumir los riesgos, un abandonarse a la suerte. Sin embargo, confiar también implica hacer frente a la posibilidad de perdida, y el temor a la pérdida genera esfuerzo y un trato creativo en las relaciones con los demás y con el mundo, trato fundamental en la satisfacción psicológica y en el descubrimiento del significado de lo moral. Si las personas no logran vivir creativamente, otorgando solidez a sus relaciones, pueden aparecer en ellas síntomas de melancolía o tendencias esquizoides que lo aparten de su principio de realidad. La confianza es pues, básica para la maduración personal y es condición necesaria para la elaboración de la auto identidad, sin ella es imposible el ajuste adecuado a un mundo de personas y de objetos y por lo tanto una existencia humana segura, y sin la confianza y la seguridad se pueden producir consecuencias traumáticas para el individuo.

Como acto creativo, éste está lleno de posibilidades no solo para una persona, sino para un grupo de perso- 
nas que estén inmersas en una relación impregnada de transparencia, seguridad y claridad de lo que se quiere y hacia donde se desea ir, es lo esperado en los jóvenes de Manizales al contar con una persona que integra su grupo.

Por eso se hace necesario que haya un aprendizaje con base en la colaboración, en la solidaridad, el apoyo, para que se pueda hacer un esfuerzo en la construcción de la asociación, ya que sin la colaboración no hay comunidad que pueda existir.

Hay que aprender a trabajar juntos, sin resquemores y sin prejuicios, es una necesidad, en la construcción de la sociedad del conocimiento (alcaborro), y no solamente allí, sino en la nueva sociedad que queremos re- construir, debemos formar y formarnos, realmente, vivir nuestro cotidiano pensando en el otro, en cómo podemos armar un equipo y proponernos salir adelante si tenemos un objetivo común. Realmente la cooperación se va volviendo en un requisito para acceder al desarrollo.

Fukuyama (1999) en su análisis y posteriormente corroborando su hipótesis, en el 2001, dice que "las naciones ricas experimentaron grandes cambios debido al quiebre en sus valores: aumentaron los crímenes, la gente perdió confianza en las instituciones y tendió a comprometerse menos y a relacionarse en grupos pequeños. Sin embargo aclara, que ésta tendencia a la atomización de la sociedad se está frenando, debido a que nuestras instintos más básicos, nos impulsan a crear reglas morales que nos unen en comunidades y a promover la cooperación."

Al no haber confianza en las instituciones, causa que la opinión pública tenga una visión negativa de los dirigentes.

\section{A MODO DE CONCLUSIÓN...}

- La familia definitivamente, tiene un papel fundamental en la transmisión generacional de los valores positivos, son el direccionamiento, el timón para las relaciones sociales futuras.

- Los sueños para los jóvenes son importantes, son el motor que hace que sus acciones se centren en esos objetivos. El pensar en un mundo mejor a partir de lo que ellos pueden modificar.

- Cambiar la realidad que viven, a partir de lo que sueñan los jóvenes como lo mejor para el mundo.
- Los jóvenes están convencidos que ellos pueden alcanzar el éxito, si desde ya tienen características de personas exitosas.

- Manejan claridad frente a una persona que tiene éxito y quién no. Siendo reiterativos en cualidades y valores positivos. En la seguridad y en la confianza que éstos proyectan.

- Son claros al plantear que lo que fortalece las relaciones en un grupo son la confianza, unido a otros valores como el respeto y la responsabilidad.

- Saben que lo que debilita al grupo es la falta de confianza entre ellos, y la falta de conocimiento de oportunidades.

- Los valores que más tienen en común son: alegría, respeto, responsabilidad, compromiso.

- Comparten conocimientos, habilidades, oportunidades, sueños, alegrías y tristezas, angustias, deseos de salir adelante y el cómo.

- Al relacionarse tienen en cuenta la escala de valores de los otros y éstos a su vez van generando la confianza y la seguridad para contar con ellos en determinados momentos.

- Se fijan en valores positivos que ayudan a potenciar al grupo.

- Al momento de pertenecer a un grupo se fijan en qué los va a beneficiar, bien sea emocionalmente, socialmente o económicamente.

- Consideran que el grupo les aporta en su forma de ser y en ir puliendo diferentes aspectos de su personalidad.

- El grupo los estimula a continuar realizando lo que allí muestra, ya sean habilidades, planes o ayudas.

- Consideran que para alcanzar a desarrollar su proyecto de vida deben tener en cuenta el ser visionarios, estrategas, estudiosos, empeño y paciencia.

- Piensan que lo que se haga debe estar impregnado de: conocimiento, amor, sentirse bien con lo que se realiza, ganas para sacar adelante lo que se quiere.

- Las comunidades de sentido las van depurando en la medida que se avanza en edad; es así como para los jóvenes de 14 a 18 , una comunidad de sentido son las subculturas (emos, rastas, rockeros, crosover, entre otros) para los de 19 a 26, las comunidades de sentido son : la familia, sus amigos, grupo musicales, redes sociales, grupos artesanales, grupos deportivos.

- Los grupos de referencia de los jóvenes son: de los de 14 a 18 personajes famosos, grupos de scout, grupo de empresarios, grupo familiar, entre otros. Para los de 18 a 26 son el grupo familiar, las orientaciones de los padres, la misma sociedad, grupo de 
voluntarios, los profesores, los técnicos, los grupos relacionados con la parte social y laboral, grupos religiosos.

- La vivencia de la ciudadanía, es otro elemento importante en el desarrollo del capital social, es ahí donde los jóvenes aprenden a vivenciar las ganancias sociales, aprenden a darse cuenta de la valía de una comunidad unida, aprende a tener objetivos comunes en un grupo y a llevarlos a cabo, aprende a vivir conforme a los códigos civiles, los cuales le proporcionarán paciencia, tolerancia y cooperación.

- Es sensato trabajar con más ahínco en todo el sector de la educación con respecto a los valores, a través del P.E.I, transversalizandolo en todos sus proyectos.

- Trabajar en nuestros jóvenes el valor de la confianza, unido al del respeto, responsabilidad, lealtad, honestidad y sinceridad.

- Estimular el aprendizaje con base en la colaboración, en la solidaridad, el apoyo, esto nos lleva a la construcción sólida de la asociatividad.

- Crear nuevas estrategias para que el joven se acerque, conozca y re-conozca en las instituciones públicas y privadas, una fuente de solidaridad y apoyo social que allí encontraría.

- Estimular y apoyar ese espíritu de entusiasmo y deseos por cambiar lo que le rodea.

- Los padres de familia deben seguir teniendo formación e información acerca del cómo enseñarles a sus hijos y como trasmitirles aquellos valores que consideran fecundos para ser unas personas exitosas, son el grupo de referencia más fuerte en todas las franjas de edades, realmente los jóvenes han vuelto a tener en cuenta que la familia es quien les proporciona y les debe proporcionar los elementos básicos para tener y mantener unas buenas relaciones sociales, y aprender a buscar redes que los puedan impulsar, con la seguridad y la confianza que desde el seno del hogar brinda, no temen así equivocarse, o buscar nuevas alianzas o alternativas.

- Se debe incrementar el nivel de confianza de los líderes y los dirigentes, para que ellos así, lo transmitan, hay que trabajar en la transparencia de los mismos, creando confianza en ellos se abre la puerta de la esperanza en un mañana mejor, en un hoy para el mañana.

- Debe facilitarse el manejo de las comunicaciones, esto ahonda y le da credibilidad a la democracia, si hay buena comunicación entre las personas y conocimiento de las mismas ellas redundarán en buenas relaciones y en conocimiento de buenas oportuni- dades para el desarrollo de proyectos. El saber expresar lo que piensan las personas, hacen que sea una característica para ser escuchado y seguido por ellos, le han dado gran importancia a la forma de expresar de las personas, no solo sus pensamientos sino también sus emociones, es darles seguridad que no solo ellos, piensan y sienten de una u otra forma.

- Todo programa que lleve inmerso el trabajo comunitario redundará en beneficios para toda una comunidad. Esto es formarnos para trabajar con la gente, para la gente y por la gente, el trabajo en equipo sin tener miramientos hacia lo que pueden pensar los otros, es tener capacidad de trabajo con el otro, aprender a reconocer que el otro también tiene habilidades, sin demeritar lo que saben o no saben hacer, es tener seguridad en lo que se es, y se tiene de ese ser

- Se debe trabajar en nuestros jóvenes y niños:

- La moral como principio básico

- El respeto a las leyes y a los reglamentos

- El respeto a los derechos de los demás

- El amor al trabajo

- La integridad

- La responsabilidad

- El orden

- La puntualidad

- El deseo de superación

- La importancia de ser un buen ciudadano

- Se debe eliminar por completo el asistencialismo, cuando una sociedad o comunidad se rige por éste principio, perderá la capacidad de lucha y búsqueda del bien común. Del potencializarse y desarrollar toda su capacidad social la cual posee. 


\section{BIBLIOGRAFÍA}

- http://edant.clarin.com/suplementos/informatica/2006/06/28/f-00411.htm. Informatica, Bacher, Silvia.

- Fukuyama, F. (1996). confianza, las virtudes sociales y la capacidad de generar prosperidad. Madrid: Editorial Atlántida.

- wwwinnovarium.com/- 2002. Conferencia en Venezuela, Fukuyama. 1999.

- Fukuyama, F. (1999). La gran ruptura. Madrid: Editorial Atlántida.

- Guiddens, Anthony. (2002). Un mundo desbocado: los efectos de la globalización. Madrid: Taurus.

- González, García Ignacio. y otros. (2002) IDEA. El país (España, Madrid) N 1140.

- Martínez, Marcelo. (2000). Crítica política:65. 2000

- www.observatorio social.com.arg.10/07/2001. Observatorio social, Asociación Civil Observatorio Social. 